\title{
Orion Absolute Navigation System Progress and Challenges
}

\author{
Greg N. Holt* and Christopher D'Souza ${ }^{\dagger}$ \\ NASA Johnson Space Center, 2101 NASA Parkway, Houston, TX, 77058
}

The Orion spacecraft is being designed as NASA's next-generation exploration vehicle for crewed missions beyond Low-Earth Orbit. The navigation system for the Orion spacecraft is being designed in a Multi-Organizational Design Environment (MODE) team including contractor and NASA personnel. The system uses an Extended Kalman Filter to process measurements and determine the state. The design of the navigation system has undergone several iterations and modifications since its inception, and continues as a work-in-progress. This paper seeks to benchmark the current state of the design and some of the rationale and analysis behind it. There are specific challenges to address when preparing a timely and effective design for the Exploration Flight Test (EFT-1), while still looking ahead and providing software extensibility for future exploration missions. The primary measurements in a Near-Earth or Mid-Earth environment consist of GPS pseudorange and deltarange, but for future explorations missions the use of star-tracker and optical navigation sources need to be considered. Discussions are presented for state size and composition, processing techniques, and consider states. A presentation is given for the processing technique using the computationally stable and robust UDU formulation with an Agee-Turner Rank-One update. This allows for computational savings when dealing with many parameters which are modeled as slowly varying Gauss-Markov processes. Preliminary analysis shows up to a $50 \%$ reduction in computation versus a more traditional formulation. Several state elements are discussed and evaluated, including position, velocity, attitude, clock bias/drift, and GPS measurement biases in addition to bias, scale factor, misalignment, and non-orthogonalities of the accelerometers and gyroscopes. Another consideration is the initialization of the EKF in various scenarios. Scenarios such as single-event upset, ground command, pad alignment, cold start are discussed as are strategies for whole and partial state updates as well as covariance considerations. Strategies are given for dealing with latent measurements and high-rate propagation using multi-rate architecture. The details of the rate groups and the data flow between the elements is discussed and evaluated.

A discussion is given on the use of a single, integrated navigation filter as opposed to several smaller filters handling separate portions of the navigation job. This is especially relevant for attitude determination and GPS measurement processing. There are many things to consider, especially when considering state data exchange, filter initializations, and extensibility.

Filter performance is affected by many factors: sensor measurement errors, data rates, tuning, and others. Foremost among these is the number and type of states that are modeled. If too many states are chosen, the filter is slow; if not enough states are modeled, the filter will perform poorly. The Orion EFT-1 Extended Kalman Filter has 11 states and 24 ECRV (Exponentially Correlated Random Variable) parameters. The parameters are modeled as first-order Gauss-Markov processes and use a much more efficient computational algorithm. The state includes attitude as a coupled member, which is needed with a strapdown IMU for initial attitude determination via gyrocompassing during pad align. It is noteworthy that the ECRV parameters for accel/gyro misalignment and nonorthogonality are only minimally observable, but are included in the filter as a more analytically correct way than process noise to condition the covariance and account for the physical presence of these known effects. A discussion is also presented on the need for separate

* Navigation Engineer, Flight Dynamics Division/Mission Operations, AIAA Senior Member

${ }^{\dagger}$ Navigation Engineer, Aerosciences and Flight Mechanics Division, AIAA Senior Member 
accelerometer biases for low-G and high-G based on the time constants of these effects. Since their computational burden is small (Gauss-Markov process), however, and there are currently no throughput issues for the $1 \mathrm{~Hz}$ EKF task, consideration is given for including all of these parameters in the filter. A future recommendation would be to allow for the use of "consider" states/parameters that allow for the benefits of noise modeling with less concern for state corruption.

A high-rate Filtered Navigator is used as the propagator complement to the EKF. The vehicle state is propagated forward in time at $200 \mathrm{~Hz}$ (the calling rate is $40 \mathrm{~Hz}$, so the input data is buffered) through the use of sensed $\Delta V$ and $\Delta \Theta$ data from the IMU. The output is used to calculate values for use by the rest of flight software. Additionally, the propagated state (position, velocity, and attitude) is sent to the EKF, where it is the primary state propagation source. The state of the Filtered Navigator is re-synched to the estimated state of the EKF at $1 \mathrm{~Hz}$ intervals via a delta state. The Orion GPS receiver utilizes a single $\mathrm{L} 1$ frequency, and is thus susceptible to measurements with ionospheric delay. Options are presented and evaluated for modeling the GPS ionosphere in different flight regimes to balance signal availability, expected delay, and filter measurement weighting. Additionally, various signal masking schema are considered. 